\title{
Morbidity in Late Preterm Birth: A Retrospective Cohort Study Assessing the Role of Immaturity versus Antecedent Factors
}

\author{
Melissa Lorenzo ${ }^{\text {a }}$ Megan Laupacis ${ }^{b}$ Wilma M.Hopman ${ }^{c}$ Imtiaz Ahmad ${ }^{d}$ \\ Faiza Khurshide \\ aDepartment of Pediatrics, University of Toronto and the Hospital for Sick Children, Toronto, ON, Canada; \\ bepartment of Pediatrics, McMaster University and McMaster Children's Hospital, Hamilton, ON, Canada; \\ 'Department of Public Health Sciences, Queen's University and Kingston Health Sciences Centre, \\ Kingston, ON, Canada; dDepartment of Biomedical and Molecular Sciences, Queen's University and \\ Kingston Health Sciences Centre, Kingston, ON, Canada; 'Department of Pediatrics, Queen's University and \\ Kingston Health Sciences Centre, Kingston, ON, Canada
}

\section{Keywords \\ Late preterm infants - Morbidity . Preterm delivery . Antecedents of delivery}

\begin{abstract}
Introduction: Late preterm infants (LPIs) are infants born between $34^{0 / 7}$ and $36^{6 / 7}$ weeks gestation. Morbidities in these infants are commonly considered a result of prematurity; however, some research has suggested immaturity may not be the sole cause of morbidities. We hypothesize that antecedents leading to late preterm birth are associated with different patterns of morbidities and that morbidities are the result of gestational age superimposed by the underlying etiologies of preterm delivery. Methods: This is a retrospective cohort study of late preterm neonates born at a single tertiary care center. We examined neonatal morbidities including apnea of prematurity, hyperbilirubinemia, hypoglycemia, and the requirement for continuous positive airway pressure (CPAP). Multivariable logistic regression analysis was performed to estimate the risk of each morbidity associated with 3 categorized antecedents of de-
\end{abstract}

livery, that is, spontaneous preterm labor, preterm premature rupture of membranes (PPROM), and medically indicated birth. We calculated the predictive probability of each antecedent resulting in individual morbidity across gestational ages. Results: 279 LPIs were included in the study. Decreasing gestational age was associated with significantly increased risk of apnea of prematurity, hyperbilirubinemia, and requirement of CPAP. In our cohort, the risk of hypoglycemia increased with gestational age, with the greatest incidence at $36^{0-6}$ weeks. There was no significant association of risk of selected morbidities and the antecedents of late preterm delivery, with or without adjustment for gestational age, multiple gestation, small for gestational age (SGA), antenatal steroids, and delivery method. Discussion and Conclusion: This study found no difference in morbidity risk related to 3 common antecedents of preterm birth in LPIs. Our research suggests that immaturity is the primary factor in determining adverse outcomes, intensified by factors resulting in prematurity.

C 2021 The Author(s) Published by S. Karger AG, Basel karger@karger.com www.karger.com/neo

Karger $\stackrel{\text { ' }}{5}$

GOPEN ACCESS
C 2021 The Author(s)

Published by S. Karger AG, Basel

This is an Open Access article licensed under the Creative Commons Attribution-NonCommercial-4.0 International License (CC BY-NC) (http://www.karger.com/Services/OpenAccessLicense), applicable to the online version of the article only. Usage and distribution for commercial purposes requires written permission.
Correspondence to:

Melissa Lorenzo, melissa.lorenzo@ sickkids.ca 


\section{Introduction}

Late preterm infants (LPIs) born between $34^{0 / 7}$ and $36^{6 / 7}$ weeks gestation account for more than three-quarters of preterm birth worldwide, representing 276,000 total births in the USA in 2018 [1, 2]. In Europe, preterm birth rates range from 5.5 to $11.1 \%$ for all live births, with 35 to 36 weeks gestation representing a median of $60 \%$ of all preterm births [3]. In a health services cost analysis during the first 3 years of life, the mean total cost of an LPI over the first 2 years of life was 2,568 Canadian dollars compared to 1,285 Canadian dollars for a term infant [4]. This relates to the increasing morbidities, such as respiratory distress and infections, requiring hospitalizations, emergency and community physician visits, and medications, in LPIs when compared to full-term infants $[4,5]$.

Late preterm delivery can occur due to spontaneous preterm labor, or it can be induced due to maternal or fetal pathologies that have rendered the intrauterine environment unsafe for the fetus. The observed morbidities in the preterm population are commonly considered a result of prematurity. However, published literature is conflicting, with some studies indicating that immaturity itself may not be the sole cause of neonatal morbidity [68]. In a study comparing LPIs and term infants exposed to similar maternal risk factors, LPIs faced significantly higher morbidities, demonstrating that adverse outcomes were a primary result of immaturity [9]. In other studies, different antecedents or underlying etiologies of late preterm delivery have been associated with varying outcomes $[6,10-13]$. As a result, it remains difficult to estimate the contribution of immaturity versus the antecedents of preterm delivery associated with morbidities in LPIs [14].

A better understanding will lead to improved triage of LPIs at birth, avoid unnecessary admissions of those who are at low risk, and provide improved anticipation of staffing needs, intensive care bed allocation, and prognosis for parents. Studies to date are limited in that they have either focused on a single morbidity or combined morbidities for risk assessment, with insufficient evaluation of outcomes at individual weeks of gestation $[9,12$, $13,15-21]$. We hypothesize that antecedents leading to late preterm birth are associated with different patterns of morbidities that are the result of gestational age superimposed by the underlying antecedents of preterm delivery.

\section{Materials and Methods}

\section{Study Population}

This is a retrospective cohort study of LPIs ( $34^{0 / 7}$ to $36^{6 / 7}$ weeks gestation) born at a single tertiary care center, Kingston Health Sciences Centre, Kingston, ON, between April 2015 and February 2017. Infants were excluded if they were outborns or if no antecedent of preterm delivery was recorded. Demographic information collected from electronically scanned paper charts included maternal medical conditions, the use of antenatal steroids, infants' sex, birth weight (grams), single versus multiple gestation, delivery method, Apgar scores, delayed cord clamping, cord arterial $\mathrm{pH}$, antecedent for preterm delivery, and length of NICU admission (days). Small-for-gestational age (SGA) status was calculated using Fenton growth charts and was defined as a birth weight $<10 \%$ for gestational age and sex.

\section{Exposures}

The antecedents of preterm delivery were determined from maternal health records. This was categorized as spontaneous prematurelabor, preterm premature rupture of membranes (PPROM), and medically indicated deliveries. Medically indicated deliveries included any maternal, fetal, or placental conditions for which the medical team decided to induce labor.

\section{Outcomes}

We used continuous positive airway pressure (CPAP) as a firstline respiratory support in infants with respiratory distress. Infants received intubation and surfactant administration if the fraction of inspired oxygen was $>0.40$ as per local guidelines. Hyperbilirubinemia and hypoglycemia were screened and managed according to Canadian Pediatric Society (CPS) Guidelines [22, 23]. CPS defined hypoglycemia as blood glucose $<2.6$ and provides guidance to initiate intravenous therapy. All babies admitted in the NICU received continuous cardiorespiratory monitoring, and apnea of prematurity was defined as clinically recorded episodes of bradycardia and desaturations $>20 \mathrm{~s}$.

\section{Data Analysis}

Baseline characteristics and neonatal outcomes were initially assessed using frequencies and percentages for categorical data, and means and standard deviations for continuous data. The Shapiro-Wilk test was used to assess the normality of the underlying distribution for continuous data. The cohort was divided into 3 groups according to (1) the antecedent of delivery - PPROM, spontaneous premature labor with intact membranes, and medically indicated birth and (2) gestational age. The Pearson $\chi^{2}$ test was used to analyze categorical values, and one-way ANOVA was used for continuous data. The Kruskal-Wallis test was performed for non-normally distributed variables such as Apgar scores and length of hospital stay. A $p$ value of less than 0.05 was considered significant, and no adjustment was made for multiple comparisons.

Simple and multivariable logistic regression modeling was used to determine the associations between exposures (antecedents of delivery) and outcomes of selected morbidities. Gestational age was converted to a continuous variable, defined by week and day (e.g., 36 $6^{2 / 7}$ would be assigned a value of 36.29 days). All morbidity outcomes, including use of CPAP, hyperbilirubinemia, hypoglycemia, and apnea of prematurity, were assessed against an- 
Table 1. Baseline characteristics of the late preterm neonates in the study according to antecedents of preterm delivery

\begin{tabular}{lccccc}
\hline Characteristic & PPROM & $\begin{array}{l}\text { Spontaneous } \\
\text { preterm labor }\end{array}$ & $\begin{array}{l}\text { Medically } \\
\text { indicated birth }\end{array}$ & Total & $p$ value \\
\hline Infants & $107(38.4)$ & $63(22.6)$ & $109(39.0)$ & 279 & $138(49.5)$ \\
Gender (male) & $57(41.3)$ & $33(23.9)$ & $48(34.8)$ & 0.383 \\
Birth weight (g) & $2,573 \pm 409$ & $2,683 \pm 511$ & $2,505 \pm 538$ & $2,571 \pm 489$ & 0.071 \\
SGA & $9(26.5)$ & $2(5.9)$ & $23(67.6)$ & $34(12.2)$ & 0.001 \\
LGA & $11(35.5)$ & $9(29.0)$ & $11(35.5)$ & $31(11.1)$ & 0.660 \\
Multiple birth & $13(31.7)$ & $6(14.6)$ & $22(53.7)$ & $41(14.7)$ & 0.105 \\
Assisted reproduction (IVF) & $6(50)$ & $1(8.3)$ & $5(41.7)$ & $12(4.3)$ & 0.445 \\
C-section & $28(24.1)$ & $15(12.9)$ & $73(62.9)$ & $116(41.6)$ & 0.001 \\
Maternal age (years) & $29.4 \pm 6.7$ & $29.0 \pm 6$ & $30.3 \pm 5.3$ & $35.4 \pm 0.86$ & 0.350 \\
Apgar score at 5 min & $8.6 \pm 1$ & $8.3 \pm 1.6$ & $8.5 \pm 1.1$ & $9 \pm 1.1$ & 0.259 \\
Delayed cord clamping & $63(42.3)$ & $34(22.8)$ & $52(34.9)$ & $149(53.4)$ & 0.257 \\
Cord pH & $7.21 \pm 0.06$ & $7.1 \pm 0.01$ & $7.2 \pm 0.05$ & $7.21 \pm 0.07$ & 0.193 \\
Antenatal steroids & $20(33.3)$ & $8(13.3)$ & $32(53.3)$ & $60(21.5)$ & 0.020 \\
Length of stay (days) & $9.0 \pm 7.9$ & $9.0 \pm 7.3$ & $9.9 \pm 7.8$ & $9.4 \pm 7.7$ & 0.680 \\
\hline
\end{tabular}

Data are presented as mean \pm standard deviation or number (percentage). PPROM, preterm premature rupture of membranes; LGA, large for gestational age; SGA, small for gestational age.

tecedents of preterm delivery. The effect of each category was estimated independently (unadjusted OR) and then adjusted for gestational age, multiple gestation, SGA, antenatal steroids, and delivery method using spontaneous preterm labor as the reference category.

\section{Results}

\section{Characteristics of the Study Population}

286 LPI infants were born during the study period. Of those, 7 infants were excluded for antecedents of delivery not documented in the chart. Therefore, 279 participants were included in the study. Table 1 demonstrates characteristics of the cohort according to the antecedents of delivery. There were 4 infants with more than one antecedent of preterm delivery, including 3 infants born due to both PPROM and spontaneous premature labor and 1 infant born due to both PPROM and a medically indicated delivery. These infants were placed in the group according to their mothers' initial presentation to hospital. Among the medically indicated births, there were 40 due to maternal hypertension (36.7\%), 24 for placental conditions $(22.0 \%), 19$ for SGA (17.4\%), 15 for maternal medical conditions (13.8\%), 7 by elective C-section (6.4\%), 3 for polyhydramnios $(2.8 \%)$, and 1 for uterine dehiscence $(0.9 \%)$. The medically indicated group was significantly more likely to receive antenatal steroids $(53.3 \%, p=$
$0.020)$, be born by C-section $(62.9 \%, p=0.001)$, and be SGA $(67.6 \%, p=0.001)$. For the PPROM group, the duration of rupture ranged from 1 to 53 days but was within 1 day for $79 \%$ of the cohort, and only 8 were beyond a 2 -week window. Nine patients in total had a pathologyconfirmed diagnosis of chorioamnionitis.

In terms of the antecedent reason for preterm delivery, the rates of delivery by spontaneous premature labor remained relatively stable at $34^{0-6}, 35^{0-6}$, and $36^{0-6}$ weeks, accounting for $23.1,33.3$, and $15.9 \%$ of deliveries, respectively. PPROM was highest at $34^{0-6}$ and $36^{0-6}$ weeks, accounting for 50.0 and $42.0 \%$ of deliveries at these gestational ages, respectively. Medically indicated deliveries increased at the later gestational ages, accounting for $26.9 \%$ of deliveries at $34^{0-6}$ weeks and 46.7 and $42.1 \%$ at $35^{0-6}$ and $36^{0-6}$ weeks, respectively.

\section{Neonatal Outcomes by Gestational Age}

Infants born at $34^{0-6}$ weeks gestation were more likely to have received antenatal steroids $(46.2 \%, p<0.001)$, have lower birth weights $(2,411 \pm 525 \mathrm{~g}, p=0.001)$, and have longer NICU admissions $(14.4 \pm 6.5$ days, $p<0.001)$ (Table 2). Infants born at $36^{0-6}$ weeks gestation were significantly more likely to be SGA $(18.3 \%, p=0.018)$ and to have received delayed cord clamping $(58.7 \%, p=0.017$ ) (Table 2). Of the entire LPI population, 36 infants required supplemental oxygen $>21 \%$, with a median of $40 \%$. Furthermore, 22 infants required surfactant and intubation. 
Table 2. Baseline characteristics of the late preterm neonates in the study according to gestational age at birth

\begin{tabular}{|c|c|c|c|c|}
\hline Characteristic & $34^{0-6}$ weeks & $35^{0-6}$ weeks & $36^{0-6}$ weeks & $p$ value \\
\hline Infants & $78(28)$ & $75(26.9)$ & $126(45.2)$ & \\
\hline Gender (male) & $39(50.0)$ & $37(49.3)$ & $62(49.2)$ & 0.978 \\
\hline Birth weight (g) & $2,411 \pm 525$ & $2,583 \pm 458$ & $2,664 \pm 462$ & 0.001 \\
\hline SGA & $5(6.4)$ & $6(8.0)$ & $23(18.3)$ & 0.018 \\
\hline LGA & $12(15.4)$ & $11(14.7)$ & $8(6.3)$ & 0.071 \\
\hline Multiple birth & $8(10.3)$ & $13(17.3)$ & $20(15.9)$ & 0.410 \\
\hline Assisted reproduction (IVF) & $4(5.1)$ & $4(5.3)$ & $4(3.2)$ & 0.701 \\
\hline C-section & $34(43.6)$ & $35(46.7)$ & $47(37.3)$ & 0.391 \\
\hline Maternal age (years) & $29.4 \pm 6.4$ & $29.8 \pm 5.4$ & $29.7 \pm 5.8$ & 0.883 \\
\hline Apgar score at $5 \mathrm{~min}$ & $8.3 \pm 1.3$ & $8.7 \pm 1.1$ & $8.6 \pm 1.2$ & 0.129 \\
\hline Cord $\mathrm{pH}$ & $7.21 \pm 0.1$ & $7.23 \pm 0.05$ & $7.21 \pm 0.06$ & 0.132 \\
\hline Delayed cord clamping & $31(39.7)$ & $44(58.7)$ & $74(58.7)$ & 0.017 \\
\hline Antenatal steroids & $36(46.2)$ & $14(18.7)$ & $10(7.9)$ & $<0.001$ \\
\hline Length of stay (days) & $14.4 \pm 6.5$ & $9.9 \pm 6.4$ & $6.0 \pm 7.4$ & $<0.001$ \\
\hline
\end{tabular}

Data are presented as mean \pm standard deviation or number (percentage). LGA, large for gestational age; SGA, small for gestational age.

Table 3. Rate of selected neonatal morbidities across gestational age at $34^{0-6}, 35^{0-6}$, and $36^{0-6}$ weeks

\begin{tabular}{llllr}
\hline Morbidity & $34^{0-6}$ weeks & $35^{0-6}$ weeks & $36^{0-6}$ weeks & $p$ value \\
\hline Hypoglycemia $(n=172)$ & $39(22.7)$ & $49(28.5)$ & $84(48.8)$ & 0.039 \\
Hyperbilirubinemia $(n=119)$ & $72(60.5)$ & $26(21.8)$ & $21(17.6)$ & $<0.001$ \\
Apnea of prematurity $(n=127)$ & $64(50.4)$ & $38(29.9)$ & $25(19.7)$ & $<0.001$ \\
CPAP $(n=70)$ & $32(45.7)$ & $21(30.0)$ & $17(24.3)$ & $<0.001$
\end{tabular}

Data presented as a number (percentage). CPAP, continuous positive airway pressure.

Table 4. Neonatal outcomes of LPIs stratified by antecedent of delivery

\begin{tabular}{|c|c|c|c|c|c|c|}
\hline Morbidity & Antecedent of delivery & $N(\%)$ & $\begin{array}{l}\text { Odds ratio unadjusted } \\
(95 \% \mathrm{CI})\end{array}$ & $p$ value & $\begin{array}{l}\text { Odds ratio adjusted } \\
(95 \% \mathrm{CI})^{*}\end{array}$ & $p$ value \\
\hline \multirow[t]{2}{*}{ Hypoglycemia } & Spontaneous preterm labor (ref) & $36(58.1)$ & & \multirow{2}{*}{0.628} & & \multirow{2}{*}{0.816} \\
\hline & Medically indicated birth & $71(65.1)$ & $1.34(0.71-2.5)$ & & $1.2(0.61-2.64)$ & \\
\hline Hyperbilirubinemia & Spontaneous preterm labor (ref) & $30(47.6)$ & & 0.101 & & 0.690 \\
\hline \multirow[t]{3}{*}{ CPAP } & Spontaneous preterm labor (ref) & $15(23.8)$ & & \multirow{3}{*}{0.399} & & \multirow{3}{*}{0.804} \\
\hline & PPROM & $23(21.5)$ & $0.87(0.41-1.83)$ & & $0.81(0.37-1.78)$ & \\
\hline & Medically indicated birth & $32(29.4)$ & $1.33(0.65-2.70)$ & & $1.00(0.43-2.81)$ & \\
\hline \multirow[t]{2}{*}{ Apnea of prematurity } & Spontaneous preterm labor (ref) & $30(47.6)$ & & \multirow[b]{2}{*}{0.893} & & \multirow[b]{2}{*}{0.468} \\
\hline & PPROM & 47 (43.9) & $0.86(0.46-1.6)$ & & $0.96(0.45-2.04)$ & \\
\hline
\end{tabular}

PPROM, preterm premature rupture of membranes; CPAP, continuous positive airway pressure; SGA, small for gestational age; LPI, late preterm infant. * Adjusted odd ratio was created using multivariate logistic regression using spontaneous preterm labor as a reference, adjusted for gestational age, multiple gestation, SGA, antenatal steroids, and delivery method. 

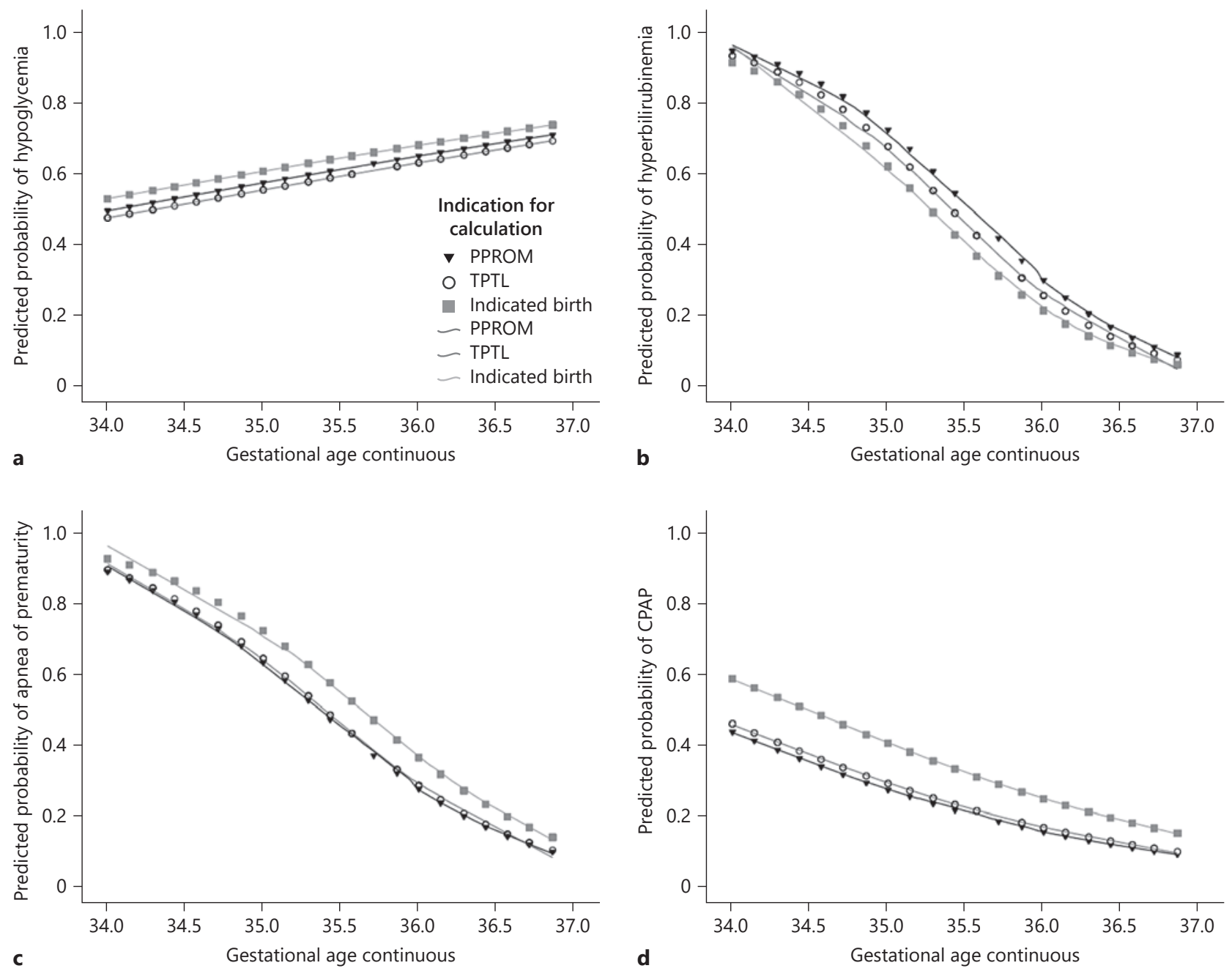

Fig. 1. Predictive risk of neonatal morbidities including hypoglycemia (a), hyperbilirubinemia (b), apnea of prematurity (c), and need for CPAP (d) by antecedents of delivery across gestational ages. CPAP, continuous positive airway pressure; PPROM, preterm premature rupture of membranes.

Decreasing gestational age was associated with significantly increased risks of apnea of prematurity $(50.4 \%, p<$ $0.001)$, hyperbilirubinemia $(60.5 \%, p<0.001)$, and the need for CPAP $(45.7 \%, p<0.001)$. The incidence of hypoglycemia was highest at $36^{0-6}$ weeks $(48.8 \%)$, versus $28.5 \%$ at $35^{0-6}$ weeks, and $22.7 \%$ at $34^{0-6}$ weeks $(p=0.039)$ (Table 3). Of the 126 infants born at $36^{0-6}$ weeks, 64 infants $(50.8 \%)$ required admission to the NICU.

\section{Neonatal Outcome by Antecedent of Delivery}

There was no significant difference in the risk of any morbidity between the antecedents of LPI delivery on the unadjusted regression models. The results remain the same after adjustment for gestational age, multiple gestation, SGA, antenatal steroids, and delivery method (Table 4).

Figure 1 shows the predictive risk of hypoglycemia, hyperbilirubinemia, apnea of prematurity, and requirement for CPAP in LPIs born by PPROM, spontaneous premature labor, and medically indicated births across gestational ages. These results were not significantly different. 


\section{Discussion and Conclusion}

This study demonstrates that LPIs born due to different antecedents of late preterm birth do not have different risk profiles in developing neonatal morbidities. In our cohort, except for hypoglycemia, the incidence of morbidities increased with decreasing gestational ages.

Data on the effect of immaturity versus antecedents of preterm delivery is conflicting. Our results confirm the findings of Shapiro-Mendoza et al. [9] demonstrating that the risks of morbidities in LPIs are due to physiological immaturity. Their study determined that LPIs exposed to similar risk factors as term infants had worse outcomes, with the independent effect of late prematurity being a 7 times greater risk factor than any of the selected maternal medical conditions.

Dimitriou et al. [24] performed a prospective study to evaluate the risk of maternal conditions on morbidities. The results were similar to our and Shapiro-Mendoza's studies in that the risk of neonatal morbidity decreases with each increasing week of gestation, reflecting maturity of the fetus [9]. Interestingly, the authors found that factors such as SGA, multiple gestation, emergency $\mathrm{C}$-section, and lack of antenatal steroid administration increased the risk of morbidities [24]. These risk factors were collected as part of demographics, rather than considered an actual maternal risk factor exposure. In our study, these factors were evaluated in the regression analysis and were not significant. Moreover, the distinction of morbidity caused by spontaneous delivery and medically indicated birth is complex and perhaps of limited significance, as 2 fetuses from 2 different pregnancies could be exposed to similar maternal medical conditions during pregnancy and mode of delivery, regardless of the antecedent of delivery [25].

Our study cohort had the same number of medically indicated births (39\%) as PPROM births (38.4\%). Typically spontaneous preterm birth is the largest group resulting in preterm delivery; however, studies generally combine PPROM and spontaneous premature labor with intact membranes and classify this as spontaneous preterm birth [26]. These numbers have been variable in different studies with spontaneous birth ranging from 17 to $49 \%[14,17,18,27,28]$. By comparing the morbidities across gestational ages, we found a significantly increased risk of apnea of prematurity, hyperbilirubinemia, need for CPAP, and longer NICU admission with decreasing gestational age. This is consistent with previous studies which have demonstrated increasing risk of morbidities with decreasing gestational ages $[9,19-21,24,25]$. In one study by Ortigosa Rocha et al. [13], a significantly increased risk of longer hospital stay and use of phototherapy was found in SGA infants. Given the majority of SGA infants found in the medically indicated delivery group, our medically indicated deliveries did not demonstrate these outcomes [13]. Interestingly, we found a significantly increased risk of hypoglycemia at $36^{0-6}$ weeks $(48.8 \%)$ compared to $28.5 \%$ at $35^{0-6}$ weeks and $22.7 \%$ at $34^{0-6}$ weeks ( $p=$ $0.039)$. This is likely explained by our center's NICU admission criteria and differences among infants in our cohort. At our center, all infants born under 36 weeks are admitted to the NICU and received targeted fluid intake either through enteral or parenteral fluid. However, infants born at 36 weeks are admitted to the postpartum ward and are only transferred to the NICU if any complications arise. Hypoglycemia is the most common reason for NICU admission at this gestation at our center. Additionally, compared to the infants born at the earlier gestational ages, infants born at $36^{0-6}$ weeks were significantly more likely to be SGA, a known risk factor for hypoglycemia [29, 30].

This study contributes to the literature of late preterm deliveries and their associated morbidities and builds on previous studies which have demonstrated that the risk of short-term morbidities in LPIs is a function of physiological immaturity interplaying with biological determinants of health acting through gestational age [8]. Raju [31] has suggested the need to develop a risk-based system incorporating both the antecedents of preterm delivery and gestational age to determine outcomes. Further determination of immaturity versus antecedent factors impacting health outcomes will help inform healthcare providers pursuing efforts to prevent or postpone preterm deliveries. Developing a risk-based approach may help with decisions surrounding the benefits or risks of early delivery for infants [6].

This study attempted to overcome limitations of previously published literature. First, we used specific outcome definitions, selecting morbidities that result in increased length of stay in the NICU, need for monitoring, and health care utilization. Most importantly, we separated threatened preterm labor and spontaneous preterm labor as antecedent factors, which have previously been combined in other studies under the umbrella of spontaneous labor [26]. Other strengths related to the use of data from a single center; the availability of individual patient records improved our ability to detect accurate maternal conditions and determine if births were a result of obstetric intervention and also provided a 
consistent method of reporting gestational age. This resulted in more accurate categorization of the antecedents of late preterm deliveries.

Despite the improved categorization, our study has limitations related to it being a single-center study with a small sample size. With single-center data, there may be a more homogenous population. The smaller sample size also has the potential to give results that do not reach statistical significance. Local practices may have also impacted our results. For example, the fact that the $36^{0-6}$ weeks infants had the highest risk of hypoglycemia may have been due in part to our NICU admission criteria. Additionally, many factors that could result in increased risk for morbidities may be distributed across the different antecedents of preterm delivery, potentially limiting the value of using antecedents for risk stratification [25]. Moreover, in this study, only short-term morbidities were examined. Assessment of long-term morbidities may have provided a different insight into associated risks.

In conclusion, our research demonstrates that the majority of LPIs suffer from morbidities resulting in hospital admission. Our results suggest that immaturity related to gestational age is the primary contributor to the major morbidities in the LPIs population. The antecedents of late preterm delivery considered in isolation are not associated with different patterns of increased neonatal morbidities. The aforementioned limitations and additional factors should be considered when discussing anticipated outcomes with parents.

\section{Acknowledgement}

The authors thank Dr. Kimberly Dow for her review of the manuscript.

\section{Statement of Ethics}

This study was approved by Queen's University Human Research Ethics Board (HSREB) (project number PAED-397-16 \#6018497). Parental consent was not required as per the HSREB as this was a retrospective study.

\section{Conflict of Interest Statement}

The authors have no conflicts of interest to declare.

\section{Funding Sources}

There are no funding sources related to this study.

\section{Author Contributions}

Melissa Lorenzo performed study design, data curation, and drafted, reviewed, and edited the manuscript. Megan Laupacis provided data curation and reviewed the manuscript. Wilma Hopan performed formal data analysis and reviewed and edited the manuscript. Imtiaz Ahmad provided data curation and reviewed the manuscript. Faiza Khurshid provided study design, methodology, supervision, data curation, resources, and drafted, reviewed, and edited the manuscript.

\section{References}

1 Raju TN. Epidemiology of late preterm (nearterm) births. Clin Perinatol. 2006 Dec;33(4): 751-vii.

2 Martin JA, Hamilton BE, Osterman MJK. Births in the United States, 2018 key findings data from the national vital statistics system. 2018 [cited 2020 May 8]. Available from: https: //www.cdc.gov/nchs/products/index.htm.

3 Zeitlin J, Szamotulska K, Drewniak N, Mohangoo AD, Chalmers J, Sakkeus L, et al. Preterm birth time trends in Europe: a study of 19 countries. BJOG. 2013 Oct;120(11):135665.

4 Bérard A, Le Tiec M, De Vera MA. Study of the costs and morbidities of late-preterm birth. Arch Dis Child Fetal Neonatal Ed. 2012 Sept;97(5):F329-34.

5 Teune MJ, Bakhuizen S, Gyamfi Bannerman C, Opmeer BC, van Kaam AH, van Wassenaer AG, et al. A systematic review of severe morbidity in infants born late preterm. Am J Obstet Gynecol. 2011 Oct;205(4):374-9.
6 Basso O. Mortality risk among preterm babies: immaturity vs. underlying pathology. Epidemiology. 2011;21(4):521-7.

7 Wilcox AJ, Weinberg CR, Basso O. On the pitfalls of adjusting for gestational age at birth. Am J Epidemiol. 2011;174(9):1062-8.

8 Brown HK, Speechley KN, Macnab J, Natale R, Campbell MK. Neonatal morbidity associated with late preterm and early term birth: the roles of gestational age and biological determinants of preterm birth. Int J Epidemiol. 2014 Jun;43(3):802-14.

9 Shapiro-Mendoza CK, Tomashek KM, Kotelchuck M, Barfield W, Nannini A, Weiss J, et al. Effect of late-preterm birth and maternal medical conditions on newborn morbidity risk. Pediatrics. 2008 Feb;121(2):e223-32.

10 Feldman K, Woolcott C, Connell CO, Jangaard K. Neonatal outcomes in spontaneous versus obstetrically indicated late preterm births in a nova scotia population. J Obstet Gynaecol Can. 2012;34(12):1158-66.
11 Lee J, Seong HS, Kim BJ, Kwan Jun J, Romero $\mathrm{R}$, Yoon BH. Evidence to support that spontaneous preterm labor is adaptive in nature: neonatal RDS is more common in "indicated" than in "spontaneous" preterm birth. J Perinat Med. 2009;37:53-8.

12 Kurkinen-Raty M, Koivisto M, Jouppila P. Preterm delivery for maternal or fetal indications: maternal morbidity, neonatal outcome and late sequelae in infants. Br J Obstet Gynaecol. 2000;107(May):648-55.

13 Ortigosa Rocha C, Bittar RE, Zugaib M. Neonatal outcomes of late-preterm birth associated or not with intrauterine growth restriction. Obstet Gynecol Int. 2010 Mar;2010: 231842.

14 Gagliardi L, Rusconi F, Da Frè M, Mello G, Carnielli V, Di Lallo D, et al. Pregnancy disorders leading to very preterm birth influence neonatal outcomes: Results of the population-based ACTION cohort study. Pediatr Res. 2013 Jun;73(6):794-801. 
15 Chen XK, Wen SW, Smith G, Yang Q, Walker M. Pregnancy-induced hypertension is associated with lower infant mortality in preterm singletons. BJOG. 2006 May;113(5): 544-51.

16 Bailit JL, Gregory KD, Reddy UM, GonzalezQuintero VH, Hibbard JU, Ramirez MM, et al. Maternal and neonatal outcomes by labor onset type and gestational age. Am J Obstet Gynecol. 2010 Mar;202(3):245-e12.

17 Laughon SK, Reddy UM, Sun L, Zhang J. Precursors for late preterm birth in singleton gestations. Obstet Gynecol. 2010;116(5):104755.

18 Gyamfi-Bannerman C, Fuchs KM, Young OM, Hoffman MK. Nonspontaneous late preterm birth: etiology and outcomes. Am J Obstet Gynecol. 2011;205(5):456-6.

19 Melamed N, Klinger G, Tenenbaum-Gavish $\mathrm{K}$, Herscovici T, Linder $\mathrm{N}$, Hod $\mathrm{M}$, et al. Short-term neonatal outcome in low-risk, spontaneous, singleton, late preterm deliveries. Obstet Gynecol. 2009 Aug;114(2 Pt 1): 253-60.
20 De Luca R, Boulvain M, Irion O, Berner M, Pfister RE. Incidence of early neonatal mortality and morbidity after late-preterm and term cesarean delivery. Pediatrics. 2009 Jun; 123(6):e1064-71.

21 JU H, Hibbard JU, Wilkins I, Sun L, Gregory K, Haberman S, et al. Respiratory morbidity in late preterm births. JAMA. 2010 Jul;304(4): 419-25.

22 Canadian Paediatric Society. Guidelines for detection, management and prevention of hyperbilirubinemia in term and late preterm newborn infants https://www.cps.ca/documents/position/hyperbilirubinemia-newborn.

23 Paediatric Society Canadian. Screening guidelines for newborns at risk for low blood glucose. Paediatr Child Health. 2004;9(10): 723.

24 Dimitriou G, Fouzas S, Georgakis V, Vervenioti A, Papadopoulos VG, Decavalas G, et al. Determinants of morbidity in late preterm infants. Early Hum Dev. 2010 Sep;86(9):58791.
25 Bailit JL, Gregory KD, Reddy UM, GonzalezQuintero VH, Hibbard JU, Ramirez MM, et al. Maternal and neonatal outcomes by labor onset type and gestational age. Am J Obstet Gynecol. 2010 Mar;202(3):245-e12.

26 Goldenberg RL, Culhane JF, Iams JD, Romero R. Epidemiology and causes of preterm birth. Lancet. 2008 Jan;371(9606):75-84.

27 Reddy UM, Ko CW, Raju TN, Willinger M. Delivery indications at late-preterm gestations and infant mortality rates in the United States. Pediatrics. 2009 Jul;124(1):234-40.

28 Holland MG, Refuerzo JS, Ramin SM, Saade GR, Blackwell SC. Late preterm birth: how often is it avoidable? Am J Obstet Gynecol. 2009;201(4):404-4.

29 Harris DL, Weston PJ, Harding JE. Incidence of neonatal hypoglycemia in babies identified as at risk. J Pediatr. 2012 Nov;161(5):787-91.

30 Hamborg-Petersen B, Hansen KN, Hansen US, Lund HT. [Hypoglycemia in the neonatal period. The need for blood sugar control]. Ugeskr Laeg. 1990 Jan;152(5):327-9.

31 Raju TNK. Moderately preterm, late preterm and early term infants: research needs. Clin Perinatol. 2013 Dec;40(4):791-7. 